\title{
Green Synthesis of Zinc Oxide Nanoparticles using Neem (Azadirachta indica) and Tulasi (Ocimum tenuiflorum) Leaf Extract and their Characterization
}

\author{
Amrutha S. Ajayan* and N. S. Hebsur \\ Department of Soil Science and Agricultural Chemistry, College of Agriculture, \\ University of Agricultural Sciences, Dharwad, 580005, Karnataka, India, \\ *Corresponding author
}

\section{A B S T R A C T}

\section{Keywords}

Green synthesis, Characterization, Neem, Tulasi, Zinc oxide nanoparticles (ZnO NPs), EDAX, SEM, PSA

Article Info

Accepted:

05 January 2020

Available Online:

10 February 2020

\begin{abstract}
Plant mediated synthesis or green synthesis of nanoparticles is gaining importance due to its simplicity, eco-friendly nature, rapid rate of synthesis, less cost and due to the disadvantages associated with chemical synthesis. The bio-active molecules present in the plant extracts are utilized for the synthesis of zinc oxide nanoparticles from its bulk molecules. A study was carried out to synthesis zinc oxide nanoparticles from neem (Azadirachta indica) and tulasi (Ocimum tenuiflorum) leaf extracts. The preliminary confirmation of synthesized nanoparticles was done by UV-spectroscopy where the absorbance peaks of synthesized zinc oxide nanoparticles were obtained in UV wavelength range (359 and $364 \mathrm{~nm}$ for neem and tulasi, respectively). The synthesized particles were characterized for their size and morphology using particle size analyzer and scanning electron microscopy. The average size (diameter) of neem and tulasi synthesized zinc oxide nanoparticles were recorded as 101.6 and $122.4 \mathrm{~nm}$, respectively in particle size analyzer (PSA). In scanning electron microcopy (SEM), ZnO nanoparticles synthesized from neem recorded flakes like or flower like morphology and size ranged from 100-300 $\mathrm{nm}$. For ZnO NPs synthesized from tulasi leaf extract recorded ovoid shape and size ranged from 70-400 $\mathrm{nm}$. Energy dispersive $\mathrm{x}$-ray analysis (EDAX) of green synthesized $\mathrm{ZnO}$ nanoparticles was done to determine its purity and chemical composition.
\end{abstract}

\section{Introduction}

Nanotechnology is a newly emerging science as well as technology which deal with the study of materials in their nano size range (1$100 \mathrm{~nm})$. Molecules are able to show some unique properties in their nano size range which can be used in different field of sciences including agriculture. Nanoparticles have already revolutionized the different field of science such as textiles, industry, information and communication technology, 
energy and electronic sector. Now focuses is on the application of nanotechnology in agriculture sector. The need of the day is to increase crop productivity from the limited natural resources to assure the food security.

Precision farming with nano-sensors, application nano based fertilizers, pesticides, herbicides, nano-clays, nano polymers etc has enhanced crop productivity from least quantity of inputs. The bioavailability of nanoparticles is more than its bulk molecules and thus use efficiency will be more for nano-formulations.

Zinc is the most widespread deficient micronutrient in the soil world over. In India, 40-42 per cent cultivated lands show Zn deficiency which is causing considerable reduction in yield. So there is need to supplement crop plants with zinc nutrient.

If the crop plants are supplied with $\mathrm{Zn}$ in their nano-formulation, the nutrient use efficiency will be more. To become economic, the production of nanoparticles should be cheap and eco-friendly.

The Green nanotechnology is arising as a new branch of nanotechnology where plant mediated synthesis of nanoparticles had already got a wide attention due to its simplicity, eco-friendly nature, rapid rate of synthesis and less cost. The chemical synthesis followed by stabilization of synthesized $\mathrm{ZnO}$ NPs cause release of toxic by-products which are harmful to the ecosystem.

Thus plant mediated synthesis or green synthesis has emerged as the best alternative to chemical synthesis. Plants are the richest sources of bio-active organic molecules which include polyphenols, flavanoids, alkaloids, terpenes, tannins, steroids, saponins etc. These phyto-chemicals are non- nutritive in nature and produced in the plants as part of their defence mechanism to tolerate any kind of stress (Tiwari et al., 2014).

Neem and tulasi are known for their medical value and are the plants which are easily available. The leaf extracts of these plants are contain organic molecules of high anti-oxidant activity which can be effectively used for the reduction of bulk molecules or larger molecules of zinc in to its nano sized particles.

Zinc nitrate, zinc carbonate, zinc sulphate etc. are some zinc bulk molecules which can be used as a precursor for the synthesis of zinc oxide nanoparticles ( $\mathrm{ZnO} \mathrm{NPs}$ ). In the present study, neem and tulasi leaf extracts were used to synthesize ZnO NPs from analytical grade zinc nitrate reagent.

\section{Materials and Methods}

\section{Green synthesis of $\mathrm{ZnO}$ NPs}

Zinc oxide $(\mathrm{ZnO})$ nanoparticles were synthesized from neem (Azadirachta indica) and tulasi (Ocimum tenuiflorum) leaf extracts using zinc nitrate $\left(\mathrm{Zn}\left(\mathrm{NO}_{3}\right)_{2}\right)$ as the precursor. The leaves were washed in running tap water to remove dirt and dust and dried under shade for 2 days.

The leaves were then oven dried at $30{ }^{\circ} \mathrm{C}$ for 6-8 days. After complete drying, the leaves were ground to powder using a mixer grinder and powdered leaf sample was stored separately in air tight polythene bags.

Ten gram powdered leaf sample was extracted with $100 \mathrm{ml}$ distilled water (1:10) and boiled for 30-45 minutes with constant stirring and filtered to get 10 per cent extract. The leaf extracts were stored in a refrigerator. Ten $\mathrm{ml}$ of 10 per cent neem and tulasi leaf extracts (in two separate beakers) was boiled on a hot water bath. 
When the leaf extract started boiling, one gram zinc nitrate was added and stirred constantly. The mixture was boiled till paste was obtained. The paste was then transferred to silica crucible and heated at high temperature of $400{ }^{\circ} \mathrm{C}$ for two hours in a muffle furnace and cooled.

Zinc oxide nanoparticles obtained as white powder were preserved in plastic vials for further characterization. The green synthesized zinc oxide nanoparticles were characterized for their size, shape and stability.

\section{Characterization of $\mathrm{ZnO}$ NPs}

\section{UV -spectroscopy}

UV-spectrophotometer analysis was done for preliminary confirmation of green synthesized $\mathrm{ZnO}$ nanoparticles. Small amount of green synthesized $\mathrm{ZnO}$ NPs was dispersed in water by ultrasonication for 30 minutes.

Dispersed sample was fed to UV- visible spectrophotometer and absorbtion peak of the sample was recorded with help of connected PC and the software SP-UV5.

\section{Particle Size Analyzer (PSA)}

Particle size analyzer was used for the proximate size determination of green synthesized ZnO NPs. Small amount of green synthesized $\mathrm{ZnO}$ NPs was dispersed in water using ultrasonication for 30 minutes.

Dispersed sample was fed to particle size analyzer to determine the size distribution of nanoparticles in the dispersed solution. From the frequency distribution curve obtained against particle size, the average size of the particles was determined with standard deviation.

\section{Scanning Electron Microscopy (SEM)}

Scanning electron microscopy of green synthesized $\mathrm{ZnO}$ nanoparticles was done to determine the morphology (size and shape) of synthesized nanoparticles. SEM analysis was done using SEI-1130 machine (at TNAU, Coimbatore).

Thin films of the sample were prepared on a coated copper grid by just placing a very small amount of the sample on the grid. Then, the film on the SEM grid was allowed to dry and the images of nanoparticles were taken to determine size and shape of nanoparticles.

\section{Energy dispersive atomic $x$-ray analysis (EDAX)}

EDAX analysis was done at TNAU, Coimbatore to determine the chemical composition of green synthesized nanoparticles. EDAX confirms the presence of zinc and oxygen, in the synthesized particles by analyzing their optical absorption.

\section{Results and Discussion}

Green synthesis and characterization of ZnO nanoparticles

Green synthesized zinc oxide nanoparticles ( $\mathrm{ZnO}$ NPs) were characterized through UVspectroscopy, particle size analyzer (PSA) and scanning electron microscope (SEM) to determine their size and shape.

\section{UV-spectroscopy}

UV-spectrophotometer analysis was done for preliminary confirmation of green synthesized nanoparticles. Absorbance peak of green synthesized $\mathrm{ZnO}$ NPs obtained in UVwavelength range (280-375 nm), which confirmed their size in nano range. 
In UV-spectroscopy, zinc oxide nanoparticles synthesized from different plant extracts showed difference in their absorbance peaks (Fig. 1 \& 2). ZnO NPs synthesized using neem extract exhibited absorbance peaks at $359 \mathrm{~nm}$ and that of tulasi was at $368 \mathrm{~nm}$.

$\mathrm{ZnO}$ NPs synthesized from neem extract exhibited their absorbtion peak at the lower wavelength was supposed to have a smaller size than that synthesized from tulasi.

When the size of bulk molecules get reduced to nano range, their absorbtion peak get shifted towards UV range from visible range. So nanoparticles exhibiting absorbtion peak at lower wavelength have smaller size than the particles exhibiting absorbtion peak at higher wavelength.

\section{Particle Size Analyzer (PSA)}

Particle size analyzer was used for the proximate size determination of green synthesized ZnO NPs, because all the particles may not have single and same dimension. It determines the size distribution of nanoparticles in its dispersed solution and average size of nanoparticles was calculated from frequency distribution curve or cumulative distribution curve along with their standard deviation.
The analytical data of particle size analyzer clearly indicated that ZnO NPs obtained with neem leaf extract was smaller than that with tulasi leaf extract. The NPs obtained with neem and tulasi recorded an average size of $101.6 \mathrm{~nm}$ (radius-50.8nm) and $122.4 \mathrm{~nm}$ (radius-61.2 nm), respectively (Fig. $3 \& 4$ ).

\section{Scanning Electron Microscope (SEM)}

Scanning electron microscopy of green synthesized $\mathrm{ZnO}$ nanoparticles determined the morphology (size and shape) of synthesized nanoparticles. $\mathrm{ZnO}$ NPs synthesized from neem extract were like nano-flakes or flowers and size ranged from 100-300 nm (Fig. 5). But that from tulasi leaf extract were spherical in shape and size ranged from 70-400 nm (Fig. $6)$.

\section{Energy Dispersive Atomic X-ray Analysis (EDAX)}

The elemental composition of green synthesized nanoparticles was determined in EDAX (Fig. 7 \& 8). ZnO NPs synthesized with neem leaf extract was found to have purity of 52 per cent and that of tulasi leaf extract was 48.8 percent. While rest of sample weight was occupied with carbon and other foreign elements (trace in amount) present in the leaf extracts.

Table.1 Chemical composition of $\mathrm{ZnO}$ nanoparticles synthesized from Neem and Tulasi leaf extract

\begin{tabular}{|c|c|c|}
\hline \multirow{2}{*}{ Composition } & $\begin{array}{l}\text { ZnO NPs synthesized from } \\
\text { neem leaf extract }\end{array}$ & $\begin{array}{l}\text { ZnO NPs synthesized from } \\
\text { tulasi leaf extract }\end{array}$ \\
\cline { 2 - 3 } & Wt \% & Wt \% \\
\hline $\mathbf{C}$ & 43.56 & 49.29 \\
\hline $\mathbf{Z n O}$ & 52.00 & 48.80 \\
\hline $\mathbf{N a}$ & 1.35 & 0.92 \\
\hline $\mathbf{A l}$ & 1.50 & 0.12 \\
\hline $\mathbf{S i}$ & 0.16 & 0.09 \\
\hline $\mathbf{S}$ & 0.51 & 0.64 \\
\hline $\mathbf{K}$ & 0.92 & 0.14 \\
\hline
\end{tabular}


Neem and Tulasi synthesized ZnO NPs had a carbon content of 43.56 and 49.29 percent respectively. The high carbon content in the synthesized $\mathrm{ZnO}$ nanoparticles provides stability to the particle in their aqueous solution. Due to the hydrophobic nature of carbon coated $\mathrm{ZnO}$ nanoparticles, it gets dispersed well in the aqueous formulation.

UV-spectrophotometer analysis was done for preliminary confirmation of green synthesized nanoparticles. Absorbance peak of bulk molecules get shifted from higher wavelength to UV-wavelength range $(280-375 \mathrm{~nm})$ when they get reduced into nano-sized particles. Absorbance peak of $\mathrm{ZnO}$ NPs synthesized from neem and tulasi leaf extract were obtained in UV-wavelength range (359 and $364 \mathrm{~nm}$, respectively), which confirmed their size in nano range (Fig. 1 and 2).

Divya et al., (2013) synthesized ZnO NPs using leaf extract of Hibiscus rosa-sinensis had their absorption peak at UV wavelength (358-375 nm) and size ranged from 40 to 56 nm. Senthilkumar and Sivakumar (2014) reported a blue shifted absorption peak of $\mathrm{ZnO}$ NPs at $325 \mathrm{~nm}$ and their average size in accordance with XRD data was $16 \mathrm{~nm}$.

Parthiban and Sundaramurthy (2015) characterized $\mathrm{ZnO}$ NPs synthesized using Pyrus pyrifolia leaf extract and obtained absorption peak at $376 \mathrm{~nm}$ and XRD pattern revealed that synthesized NPs were having wurtzite hexagonal structure with an average size $22 \mathrm{~nm}$. Bala et al., (2015) synthesized $\mathrm{ZnO}$ NPs from Hibiscus subdariffa which showed its absorbance peaks at $370 \mathrm{~nm}$ and recorded dumbbell shape with $30-50 \mathrm{~nm}$ as diameter.

Particle size analyzer was used for the proximate size determination of synthesized $\mathrm{ZnO}$ NPs, because all the particles may not have single and same dimension.
It determines the size distribution of nanoparticles in its dispersed solution and average size of nanoparticles was calculated from frequency distribution curve (Fig. 3 and 4).

In particle size analyzer radius of $\mathrm{ZnO}$ NPs synthesized from neem and tulasi leaf extract were recorded as 50.8 and $61.2 \mathrm{~nm}$, respectively (diameter- 101.6 and $122.4 \mathrm{~nm}$, respectively).

Scanning electron microscopy (SEM) of synthesized $\mathrm{ZnO}$ nanoparticles was done to determine its morphology (size and shape). In SEM, ZnO nanoparticles synthesized from neem recorded shape as flakes like or flower like and size ranged from 100-300 nm (Fig 5).

For $\mathrm{ZnO}$ NPs synthesized from tulasi, shape was recorded as ovoid with size ranged from 70-400 nm (Fig. 6). FTIR spectroscopy of synthesized $\mathrm{ZnO}$ NPs showed presence of hydroxyl, aromatic, amine and carbonate group indicating that these functional groups from leaf extracts were responsible for the synthesis of $\mathrm{ZnO}$ NPs (Poovizhi and Krishnaveni, 2015). Sindhura et al., (2013) synthesized $\mathrm{ZnO}$ NPs using leaves of Parthenium hysterophorous and recorded size ranging from 16 to $108.5 \mathrm{~nm}$ and shape was spherical.

Gnanasangeetha and Thambavani (2014) reported neem mediated synthesis of $\mathrm{ZnO}$ NPs and obtained particles of flower morphology with size range 100-200 nm and for Oudhia et al., (2015) ZnO NPs synthesized using neem leaf extract was tubular shape with size $25 \mathrm{~nm}$. Raut et al., (2013) synthesized ZnO NPs from tulasi and obtained hexagonal shape nanoparticle with diameter range 11-25 nm. 
The elemental composition of green synthesized nanoparticles was determined in EDAX (Fig. 7 and 8). It was found that $\mathrm{ZnO}$ NPs synthesized with neem leaf extract has 52 and 43.56 percent $\mathrm{ZnO}$ and carbon, respectively, while that from tulasi leaf extract found to have 48.8 and 49.29 percent $\mathrm{ZnO}$ and carbon, respectively.

While rest of the sample weight was because of other elemental impurities from leaf extracts. Bala et al., (2015) reported the presence of carbon and other elemental

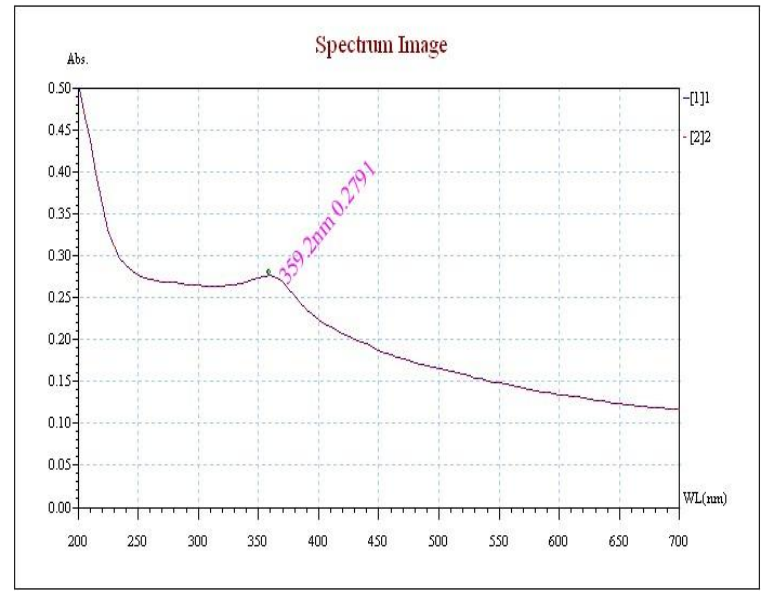

Fig.1 Absorbtion peak of $\mathrm{ZnO}$ NPs synthesized from neem leaf extract in UVSpectroscopy

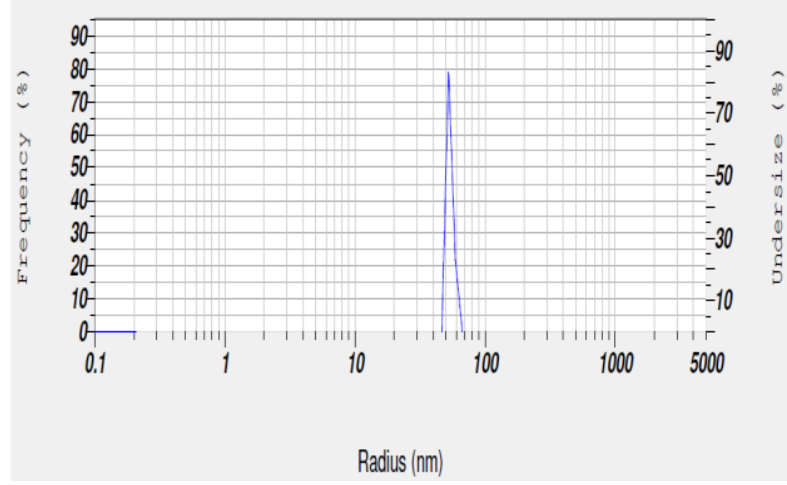

Fig.3 Average radius of ZnO NPs synthesized from neem leaf recorded in particle size analyser impurities in small amount and concluded that the stability of green synthesized nanoparticles could be due to organic molecules.

Dispersed aqueous solution of green synthesized nanoparticles showed better stability due to the presence of organic molecules. Hydrophobic nature of organic molecules prevents agglomeration of nanoparticles and causes its effective dispersion and stabilization in aqueous solution.

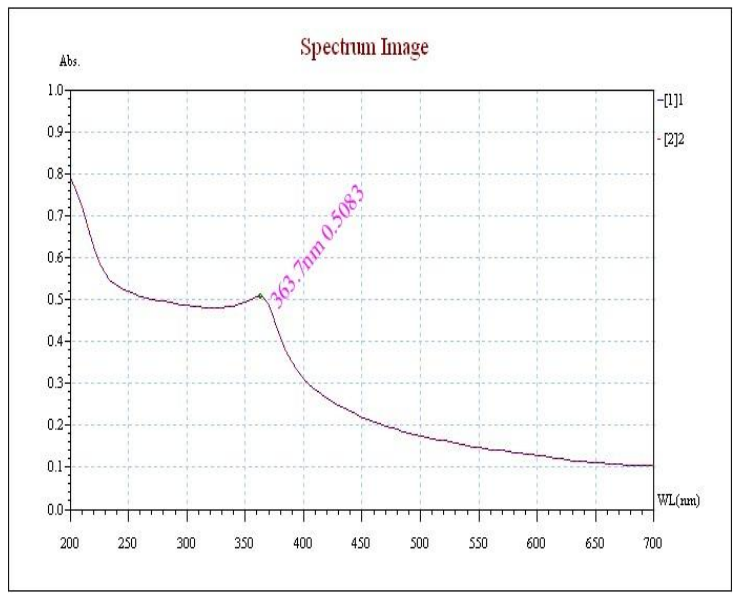

Fig.2 Absorbtion of Peak of ZnO NpPs. synthesized from tulasi leaf extract in UVSpectroscopy

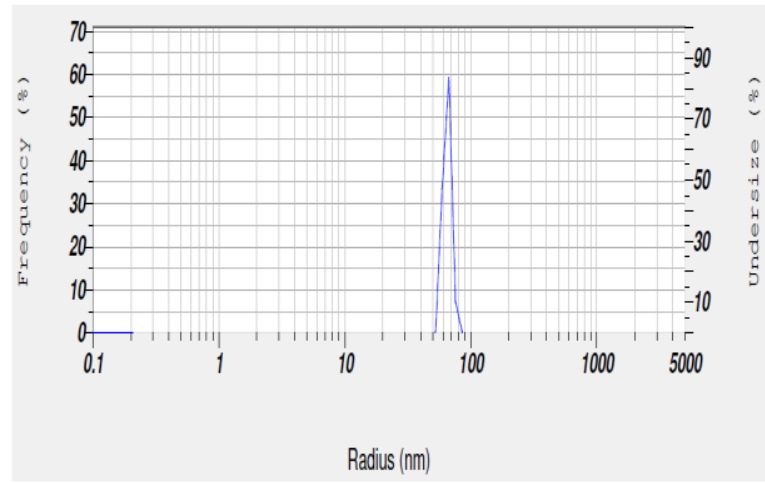

Fig.4 Average radius of $\mathrm{ZnO}$ NPs synthesized from tulasi leaf recorded in particle size analyser 


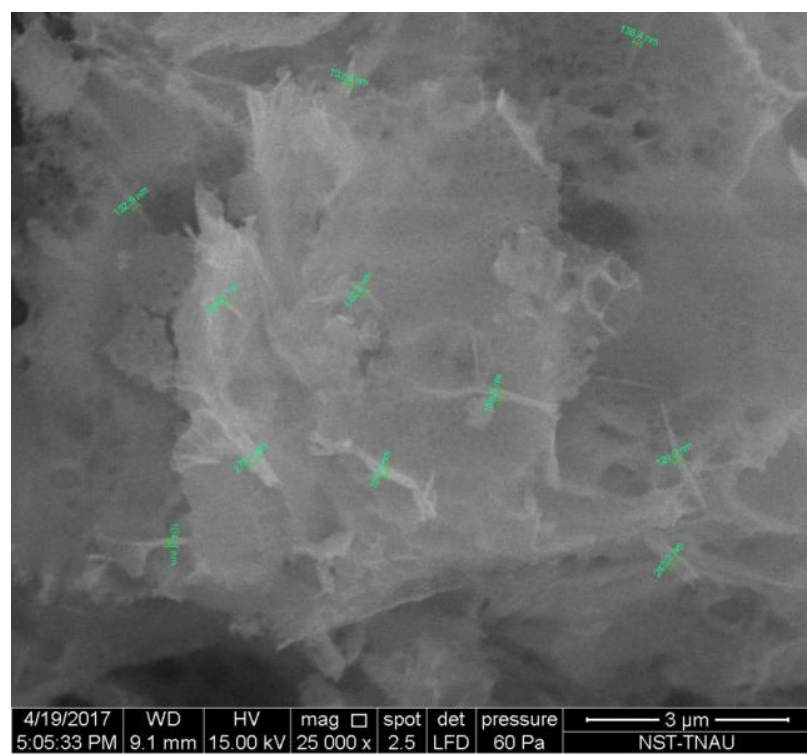

Fig.5 SEM image of ZnO NPs synthesized from neem leaf extract

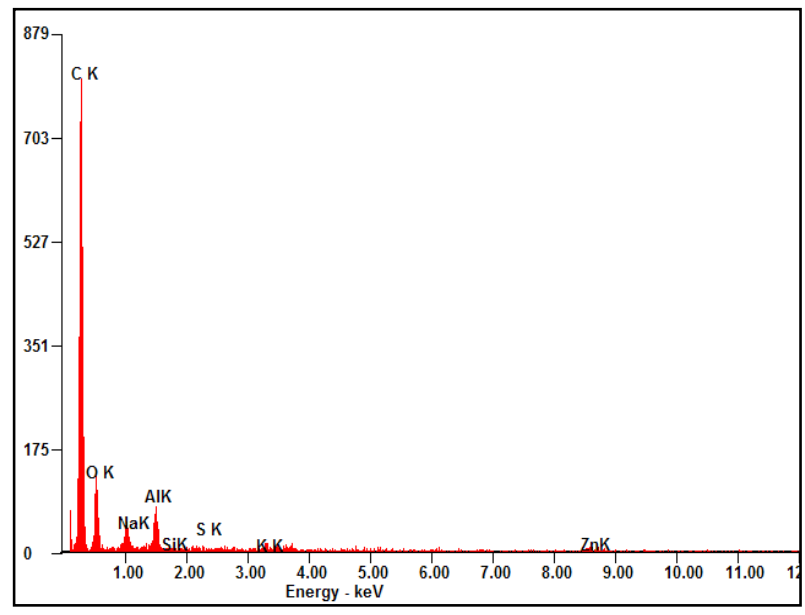

Fig.7 EDAX analysis of ZnO NPs synthesized from neem leaf extract

$\mathrm{ZnO}$ nanoparticles can be green synthesized from analytical grade reagent zinc nitrate using the leaf extract of different plants. Here for the present study, neem and tulasi leaf extract were used. The two plants are known for their medicinal values which are contributed by the active principle and biomolecules present in their extract.

The bio-molecules present in the leaf extract

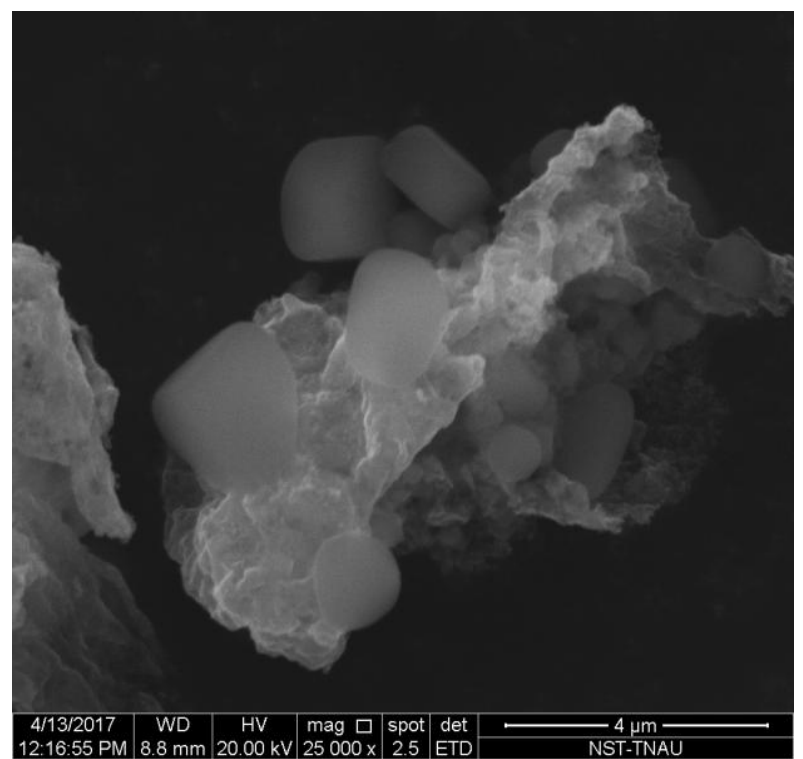

Fig.6 SEM image of ZnO NPs synthesized from tulasi leaf extract

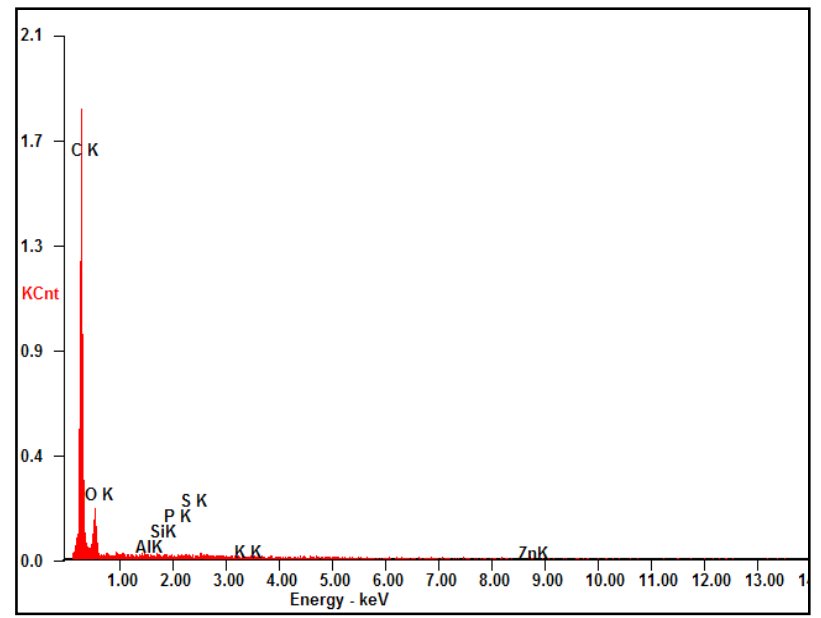

Fig.8 EDAX analysis of ZnO NPs synthesized from tulasi leaf extract

acts as reducing, capping and stabilizing agent and convert bulk molecules into its nanosized particles. The chemical synthesis and stabilization of $\mathrm{ZnO}$ NPs cause release of toxic by-product which is harmful to the ecosystem.

Also during chemical synthesis some toxic chemicals get adsorbed on the surface of $\mathrm{ZnO}$ NPs which restrict its medical applications. 
Some chemical synthesis methods require high temperature and pressure which make the synthesis process expensive. Presently, plant mediated synthesis or green synthesis has emerged as the best alternative to chemical synthesis of nanoparticles due to their simplicity, cheapness and eco-friendly nature.

These green synthesized nanoparticles were found to be more stable than the chemically synthesized nanoparticles due its high carbon content which make it hydrophobic in nature. Thus these particles get dispersed well aqueous solution and remain stable for a longer period and are suitable for preparing aqueous formulation.

Foliar application $\mathrm{ZnO}$ NPs at low concentration (250-750 ppm) is found to be effective increasing dry matter and yield maize plants (Chaitra, 2015). Green synthesized $\mathrm{ZnO}$ NPs can be used as an efficient zinc source to supplement crop plants grown in $\mathrm{Zn}$ deficient areas.

\section{Acknowledgement}

The authors gratefully acknowledge the Department of Nano Science \& Technology, Tamil Nadu Agricultural University, Coimbatore for their technical support for the accomplishment of research work

\section{References}

Bala, N., Saha, S., Chakraborty, M., Maiti, M., Das, S., Basub, R. and Nandy, P., 2015. Green synthesis of zinc oxide nanoparticles using Hibiscus subdariffa leaf extract: effect of temperature on synthesis, antibacterial activity and anti-diabetic activity. RSC Adv., 5: 4993-5003.

Chaitra, S. P., 2015, Studies on nano zinc oxide dynamics and response of maize to foliar spray of nano formulations in a Vertisol under controlled condition. M. Sc. Thesis, Univ. Agric. Sci., Dharwad, Karnataka (India).

Divya, M. J., Sowmia, C., Joona, K. and Dhanya, K. P., 2013. Synthesis of zinc oxide nanoparticle from Hibiscus rosa-sinensis leaf extract and investigation of its antimicrobial activity. Res. J. Pharm. Biol. Chem. Sci., 4 (2): 1137-1142.

Gnanasangeetha, D. and Thambavani, S. D., 2014. Facile and eco-friendly method for the synthesis of zinc oxide nanoparticles using Azadirachta and Emblica. Int. J. Pharm. Sci. Res., 5 (7): 2866-2873.

Oudhia, A., Kulkarni, P. and Sharma, S., 2015. Green synthesis of $\mathrm{ZnO}$ nanotubes for bioapplications. Int. J. Adv. Eng. Res. Studies, 4 (2): 280281.

Parthiban, C. and Sundaramurthy, N., 2015. Biosynthesis, characterization of $\mathrm{ZnO}$ nanoparticles by using Pyrus pyrifolia leaf extract and their photocatalytic activity. Int. J. Innov. Res. Sci., 4 (10): 9710-9718.

Poovizhi, J. and Krishnaveni, B., 2015. Synthesis, characterization and antimicrobial activity of zinc oxide nanoparticles synthesized from Calotropis procera. Int. J. Pharm. Sci. Drug Res., 7 (5): 425-431.

Raut, S., Thorat, P. V. and Thakre, R., 2013. Green synthesis of zinc oxide $(\mathrm{ZnO})$ nanoparticles using Ocimum tenuiflorum leaves. Int. J. Sci. Res., 5: 1225-1228.

Sabir, S., Arshad, M. and Chaudhari, S. K., 2014. Zinc oxide nanoparticles for revolutionizing agriculture: synthesis and applications. Sci. World J., 10: 63-68.

Senthilkumar, S. R. and Sivakumar, T., 2014. Green tea (Camellia sinensis) 
mediated synthesis of zinc oxide $(\mathrm{ZnO})$ nanoparticles and studies on their antimicrobial activities. Int. J. Pharm. Sci., 6 (6): 461-467.

Sindhura, K. S., Prasad, T. N. V. K. V., Selvam, P. and Hussain, O. M., 2013. Synthesis, characterization and evaluation of effect of phytogenic zinc nanoparticles on soil exo-enzymes. Appl. Nanosci., 4: 819-827.
Tiwari, D. K., Schubert, N. D., Cendejas, L. M. V., Villegas, J., Montoya, L. C and Garcia, S. E. B., 2013. Interfacing carbon nanotubes (CNT) with plants: enhancement of growth, water and ionic nutrient uptake in maize (Zea mays L.) and implications for nanoagriculture. Appl. Nanosci., 4: 577-591.

\section{How to cite this article:}

Amrutha S. Ajayan and Hebsur. N.S. 2020. Green Synthesis of Zinc Oxide Nanoparticles using Neem (Azadirachta indica) and Tulasi (Ocimum tenuiflorum) Leaf Extract and their Characterization. Int.J.Curr.Microbiol.App.Sci. 9(02): 277-285.

doi: https://doi.org/10.20546/ijcmas.2020.902.035 